\title{
Mitigating Quantization Lobes in mmWave Low-bit Reconfigurable Reflective Surfaces
}

\author{
Bharath G. Kashyap, Student Member, IEEE, Panagiotis C. Theofanopoulos, Student Member, IEEE, \\ Yiran Cui, Student Member, IEEE, and Georgios C. Trichopoulos, Member, IEEE
}

\begin{abstract}
We present a method for the mitigation of quantization lobes in single-bit reconfigurable reflective surfaces (RRSs). Typically, RRSs are planar beamforming structures consisting of hundreds or thousands of antennas with integrated tunable switches. Under plane-wave illumination, single-bit RRSs suffer from undesired side lobes or quantization lobes, which are caused by the periodicity of the errors due to the limited number of bits used in phase quantization. In this work, we present a topology that suppresses the quantization lobes using single-layer, 1-bit RRSs, by implementing a fixed but random phase delay in every unit-cell. The introduction of phase randomization breaks the periodicity of the quantization errors, thus reducing the quantization lobe level (QLL). We carry out a theoretical analysis to demonstrate the effect of phase randomization in RRSs, and for the first time, provide the condition for choosing the range of randomization required to achieve the lowest sidelobe level (SLL). Leveraging this condition, we design a single-layer, 1-bit $30 \times 30$ randomized RRS at $222.5 \mathrm{GHz}$. The reflective surface is fabricated on a thin, low-loss alumina ribbon ceramic wafer from Corning Inc. using a simplified fabrication technique suitable for largescale production of mmWave/THz RRSs. Finally, we present the radar cross-section (RCS) characterization results obtained from a quasi-optical measurement setup validating the mitigation of quantization lobes using the proposed randomization technique.
\end{abstract}

Index Terms - reconfigurable reflective surface, mmWave, quantization lobe, intelligent reflective surface

\section{INTRODUCTION}

$\mathrm{T}$ HE small wavelengths of millimeter-wave (mmWave) and terahertz (THz) frequencies allow for exciting beamforming applications with very narrow beamwidths while maintaining moderately sized apertures. As such, numerous mmWave/THz applications have been developed in these frequencies, including wireless communications, radar imaging, remote sensing, and space exploration

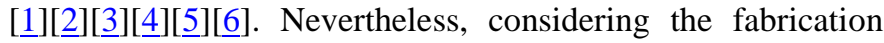
complexities and the material performance drop that occur in the mmWave/THz bands, it is an engineering challenge to implement low-cost apertures with low-loss beamforming capabilities.

At microwave frequencies, beam reconfigurability is typically realized by leveraging analog and digital phased array antennas [7][]. In analog phased arrays, switch-based phase

This paragraph of the first footnote will contain the date on which you submitted your paper for review. This work is partly supported by the National Science Foundation under Grant CAREER ECCS-1847138. shifters are integrated into the RF-front-end, and the phases of the individual antenna elements are modulated, thereby enabling beamforming and steering. On the other hand, in digital phased arrays, each antenna is connected to a mixer and an analog-to-digital converter. Digital post-processing is employed to achieve beamforming and steering. However, in both topologies, each radiating element is connected to a series of radiofrequency (RF) devices, which lead to a large hardware footprint, high power consumption, increased losses, and consequently a higher implementation cost [] $]$.

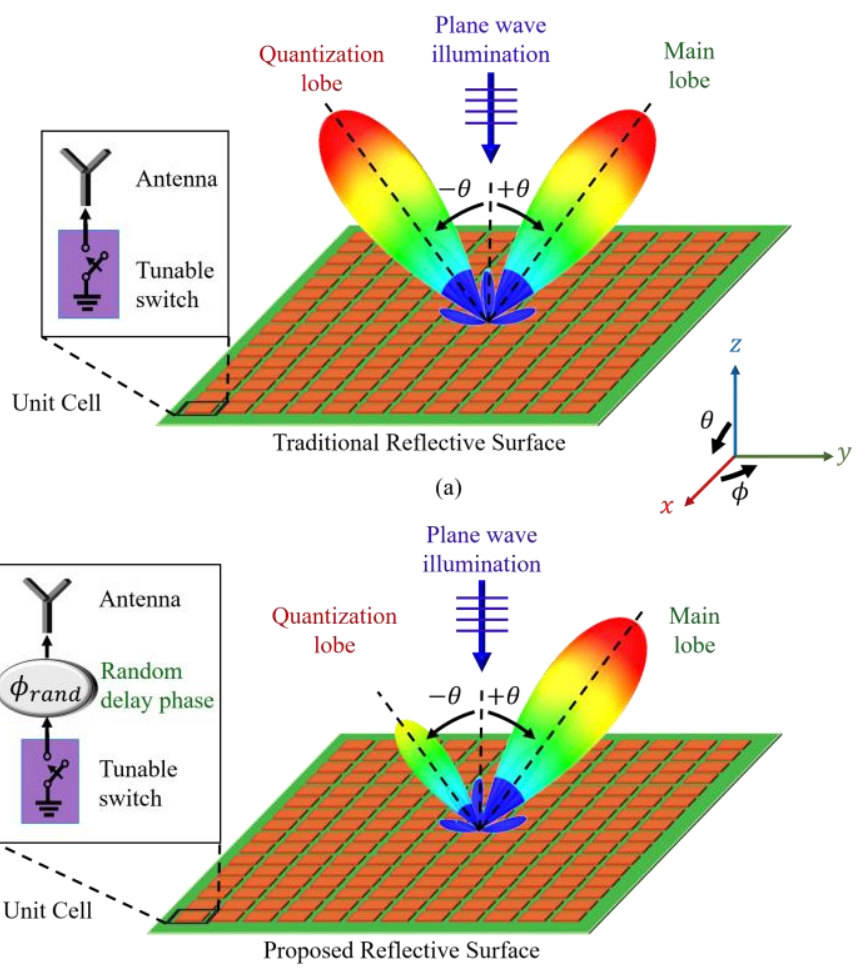

(b)

Fig. 1. Beamsteering with (a) 1-bit non-randomized RRS results in parasitic quantization lobe. Adding random phase delays in the unit-cells (b) suppresses the undesired lobe.

On the other hand, reconfigurable reflective surfaces (RRSs) that exhibit a low-profile, low-RF-losses, low-powerconsumption, and high-gain capabilities represent viable candidates for future high-frequency wireless communication applications [9]. As such, in RRSs, the absence of RF chain in

The authors are with the Ira A. Fulton School of Engineering, Arizona State University, Tempe, AZ 85287, USA. (e-mail: Bharath.kashyap@asu.edu, ptheofan@asu.edu, ycui36@asu.edu, gtrichop@asu.edu). 
the feed network of each radiating element reduces the power consumption, hardware complexity as well as RF-losses, thus bringing down the implementation cost of the RRSs [10], while enhancing overall efficiency []]. Most commonly, RRSs are fed by horn antennas to form reflectarray antennas [11][12][13]. Recently, there has been an added interest towards intelligent reflective surfaces (IRSs), where an RRS is integrated with a wireless communication system to improve signal coverage or enable new types of sensing [14][15]. In such installations, an RRS is not fed from a fixed feed as in reflectarray antennas but can receive waves from multiple angles and redirect the reflected signals to various directions depending on the application. As such, RRSs can act as relays in mmWave/THz communication systems to improve the wireless channel between users and the base station. One such typical RRS deployed as an IRS is shown in Fig. 1a. Its unit-cell consists of a patch antenna embedded with a tunable switch to realize the phase modulation. The tunability can be achieved using PIN diodes [10][11] or graphene patches [16].

Ideally, by applying a series of continuous progressive phase shifts, the main reflected beam can be steered to the desired direction. However, the implementation of continuous phase shifts significantly increases the design complexity or requires continuous phase modulators, such as varactors, that exhibit very poor performance at mmWaves and above [17]. As such, the continuous phases are usually quantized by rounding them off to discrete values through 1-, 2-, or 3-bit quantization schemes [18]. The 1-bit quantization scheme is commonly used owing to its design simplicity and lower implementation cost when compared to the higher-bit quantization schemes. However, under plane wave illumination, the quantized coding techniques introduce grating lobes due to the periodicity of the errors resulting from the limited number of bits used in the phase rounding quantization [19]. These undesired lobes or quantization lobes appear in addition to the main lobe, as shown in Fig. 1a. The issue is particularly aggravated in a 1-bit quantization scheme where the quantization lobe has the same power as the main lobe. Thus, to exploit the advantages of a 1bit quantization scheme, it is critical to break the periodicity of the phase rounding quantization error and suppress the quantization lobes.

Accordingly, herein, we present a technique to mitigate the quantization lobes, in planar, single-layer 1-bit RRSs, that appear when illuminated with plane waves as in the IRS systems. Specifically, we adopt the principles of random phasing originally proposed in multi-bit phased array systems [19] to break the periodicity of the phase rounding quantization error and thereby mitigate the quantization lobes in RRSs. The technique is realized by incorporating a randomized phase delay in each unit-cell of the RRS, as shown in Fig. 1b. A similar procedure was recently employed to mitigate the quantization lobes in 1-bit designs using a two-layer reflective metasurface excited by normally incident plane waves at 39 $\mathrm{GHz}$ [20]. A stacked-layer implementation with two substrate layers and three metal layers is employed with one metal layer acting as the tunable antenna element and the other as the delay. However, such multi-layer implementation can result in increased design complexity and possibly more losses when scaling to higher frequencies. With multiple dielectric layers, the fabrication complexity also increases due to misalignment between layers, airgaps, and bows and/or warpages in the individual wafers.

In this work, we present a technique to mitigate quantization lobes by introducing random phase delays in a single layer RRS with 1-bit quantization. Some of the preliminary simulation results were presented in [21], where a 20-element linear RRS is designed on a $20 \mu \mathrm{m}$ high-resistivity $\mathrm{Si}\left(\epsilon_{r}=11.9\right)$ at 275 GHz. Herein, we extend the study by focusing on the design, fabrication as well as characterization of the 2D RRS on an alumina ribbon ceramic wafer $\left(\epsilon_{r}=10\right.$, $\left.\tan \delta=0.8 \times 10^{-3}\right)$ at $222.5 \mathrm{GHz}$.

The rest of the paper is organized as follows: We start by detailing the technique of random phasing used for mitigating quantization lobes Section II. Then we present the design and simulation results of the proposed $30 \times 30$ reflective surface in Section III. It is followed by Section IV, which outlines the fabrication methodology suitable for large scale manufacturing of sub-mmWave/THz RRSs. Subsequently, the quasi-optical measurement setup developed for characterizing the RCS at sub-mmWave frequencies is presented in Section $\mathrm{V}$ along with the measurement results. We then carry out a discussion on the efficiency of the proposed RRS, along with the associated loss mechanisms in Section VI. Finally, we present the conclusions of this work in Section VII.

\section{Mitigating Quantization Lobes Using RANDOM PHASING}

In this section, we present a comprehensive study of the random phasing technique employed to suppress the quantization lobes appearing in 1-bit RRS designs.

\section{A. Theory of Random Phasing}

Let us consider a two-dimensional planar 1-bit RRS consisting $\mathrm{M} \times \mathrm{N}$ radiating elements arranged on the $x-y$ plane and illuminated by a plane wave from $\left(\theta_{i}, \phi_{i}\right)$ direction. To steer the reflected main beam to the desired direction $\left(\theta_{d}, \phi_{d}\right)$, the excitation phase induced on the currents of the $m n^{\text {th }}$ element is given by

$$
\Phi_{m n}=\Phi_{m n}^{\mathrm{o}}-\Phi_{m n}^{\text {illum }}
$$

where $\Phi_{m n}^{o}$ is the phase delay required by the reflective elements to deflect the main beam in the $\left(\theta_{d}, \phi_{d}\right)$ direction when the RRS is illuminated from broadside $\left(\theta_{i}=0^{0}\right)$. $\Phi_{m n}^{\text {illum }}$ is the phase distribution of the element currents induced by a plane wave impinging from $\left(\theta_{i}, \phi_{i}\right)$. These phase distributions are given by [8]

$$
\begin{gathered}
\Phi_{m n}^{\mathrm{o}}=-k_{0}\left(x_{m} \sin \theta_{d} \cos \phi_{d}+y_{n} \sin \theta_{d} \sin \phi_{d}\right) \\
\Phi_{m n}^{\text {illum }}=k_{0}\left(x_{m} \sin \theta_{i} \cos \phi_{i}+y_{n} \sin \theta_{i} \sin \phi_{i}\right)
\end{gathered}
$$

where $k_{0}$ is the free space wavenumber and $\left(x_{m}, y_{n}\right)$ represents the row and column indices of the $m n^{\text {th }}$ element.

Considering the 1-bit quantization scheme, all the phase values are rounded off to $0^{\circ}$ or $180^{\circ}$, and the periodicity of the errors resulting from this quantization scheme leads to the generation of quantization lobes in traditional RRSs. To suppress such lobes, we add random phase delays, $\Phi_{m n}^{\text {rand }}$ at 
every unit-cell using physical delay lines. Thus, the continuous excitation phase of the $m n^{\text {th }}$ element given by (1) becomes

$$
\Phi_{m n}=\Phi_{m n}^{\mathrm{o}}-\Phi_{m n}^{i l l u m}-\Phi_{m n}^{\text {rand }}
$$

The random phase delays in (4) are obtained from a uniform pseudorandom number generator using

$$
\Phi_{M \times N}^{r a n d}=\operatorname{rand}(M, N) \cdot 180^{\circ}
$$

where, $\Phi_{M \times N}^{r a n d}$ is the $\mathrm{M} \times \mathrm{N}$ matrix of random phases. A uniform distribution is considered in this work to generate random phase delays and not a gaussian or a triangular distribution as the former gives the maximum achievable SLL when compared to the latter two distributions [19]. As such, the resultant quantized phase delay of each RRS element is a binary $\left\{0^{\circ}, 180^{\circ}\right\}$ distribution:

$$
\Phi_{m n}^{q}=\mid 180^{\circ} \cdot \text { floor }\left(\frac{\Phi_{m n}}{180^{\circ}}+0.5\right) \mid
$$

All the phase values between $\left[-90^{0}, 90^{\circ}\right]$ are assigned to $0^{\circ}$ (state " $0 " / \mathrm{OFF}$ ), and the remaining values are assigned to $180^{\circ}$ (state "l"/ON). Consequently, the array factor of the randomized RRS is given by

$$
A F_{R R S}(\theta, \phi)=\sum_{i=1}^{m} \sum_{j=1}^{n} e^{-j \Phi_{m n}^{\text {total }}} e^{-j k_{0}\left(x_{m} u+y_{n} v\right)}
$$

where $\Phi_{m n}^{\text {total }}=\Phi_{m n}^{\mathrm{q}}+\Phi_{m n}^{\text {illum }}+\Phi_{m n}^{\text {rand }}$ corresponds to the total phase profile of each radiating element, $k_{0}\left(x_{m} u+y_{n} v\right)$ corresponds to the phase modulation due to Green's function, $u=\sin \theta \cos \phi$, and $v=\sin \theta \sin \phi$.

\section{B. Effect of Random Phase Delays}

To understand the effect of random phase delays in RRS designs, a 30-element linear 1-bit RRS under broadside plane wave illumination $\left(\Phi_{M \times N}^{\text {illum }}=0\right)$ is considered. Without randomization $\left(\Phi_{M \times N}^{r a n d}=0\right)$, quantization errors due to phase rounding exhibit a periodic distribution across the RRS, leading to a periodic $\Phi_{M \times N}^{q}$ profile, as shown in Fig. 2b. This spatial wave component results in a secondary lobe, as shown in the radiation pattern of Fig. 3a. For the 30-element RRS example considered here, the main lobe is designed to be at $-30^{\circ}$, while the undesired quantization lobe appears at $+30^{\circ}$. In other words, (7) gives the same phase modulation for dominant radiation at $-30^{\circ}$ as well as $+30^{\circ}$. In general, under plane wave illumination, the quantization lobe level (QLL) is equal in magnitude to the main lobe and appears at a direction symmetrically opposite to that of the specular reflection angle $\left(\theta_{i}=0^{0}\right.$ for this example).

When a small range of randomization is introduced, for example, $0-60^{\circ}$, then $\Phi_{M \times N}^{r a n d} \neq 0$, the random phase delays obtained from (5) are between 0 and $60^{\circ}$, as shown in Fig. 2c. Such a phase range is insufficient to break the periodicity of the quantization error. As such, the errors maintain a quasi-periodic distribution in $\Phi_{M \times N}^{q}$, as shown in Fig. 2d. This is significantly more pronounced in smaller arrays due to a limited number of elements, and thus, there is only a slight reduction in the QLL $(-0.89 \mathrm{~dB})$, as shown in Fig. 3b. On the other hand, as the range of randomization is increased to $0-120^{\circ}$, there is a noticeable change in the phase distribution that results in the QLL reduction by $3.61 \mathrm{~dB}$, as depicted in Fig. 2e, 2f, and $3 \mathrm{c}$ respectively.

Finally, when the full range of randomization $\left(0-180^{\circ}\right)$ is utilized to randomize the coding scheme, as shown in Fig. $2 \mathrm{~g}$ and $2 \mathrm{~h}$, the quantization lobe reaches a minimum value $(\mathrm{QLL}=-11.53 \mathrm{~dB})$. Table I summarizes the SLLs achieved in 30- and 200-element linear RRS and 30×30 2D RRS.

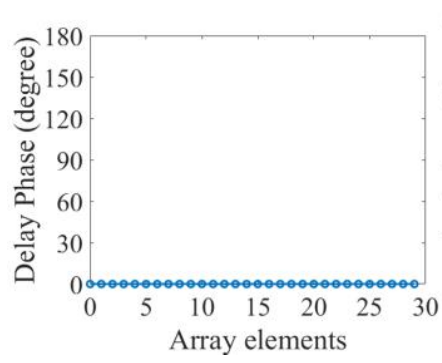

(a)

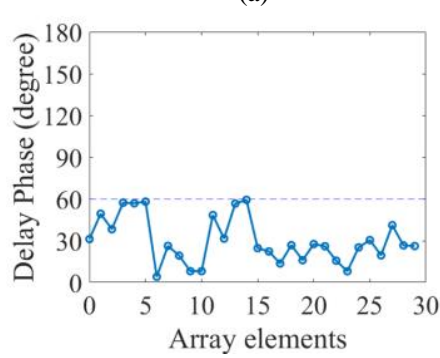

(c)

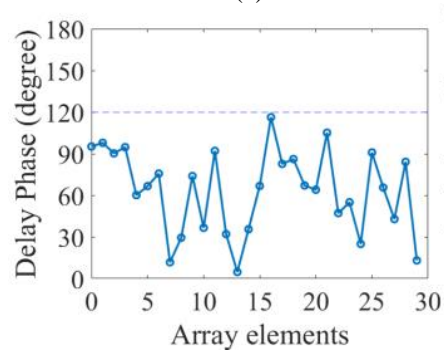

(e)

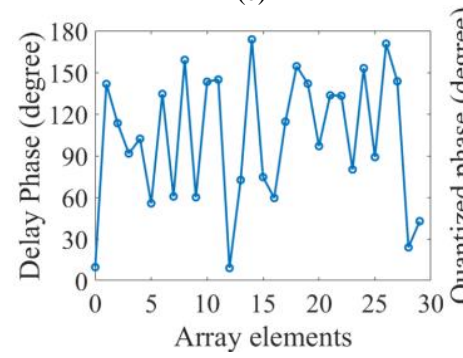

(g)

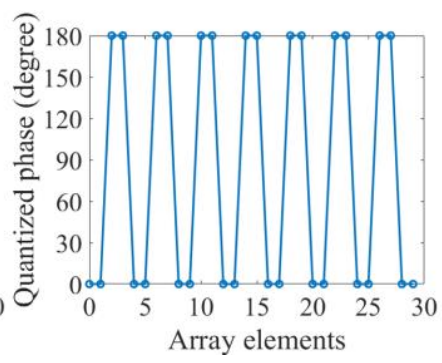

(b)

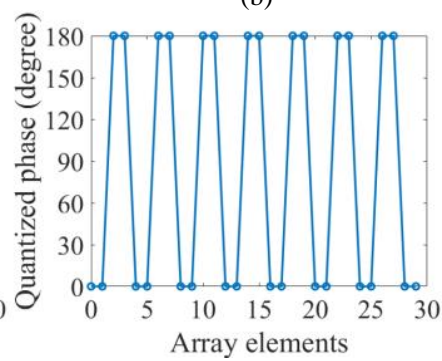

(d)

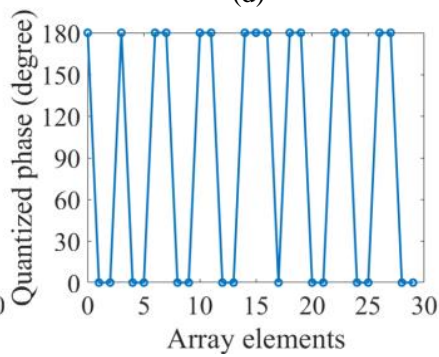

(f)

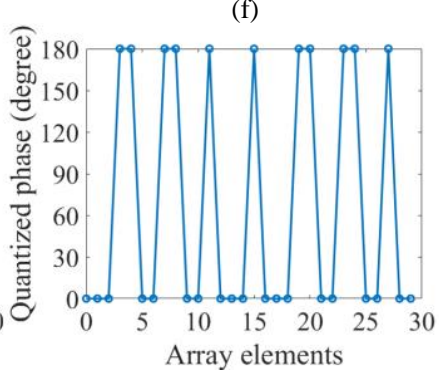

(h)
Fig. 2. Randomly generated delay phases with varying range of randomization (left) and corresponding quantized binary phases (right) of 30-element (linear) 1-bit RRS. (a), (b) $0^{\circ}$ (no randomization), (c), (d) randomization range of $0-60^{\circ}$, (e), (f) $0-120^{\circ}$, and (g), (h) $0-180^{\circ}$.

To understand the effect of the range of phase delays on the acquired SLL, a comparison is carried out for apertures of various sizes and bit configurations. As such, the results of the analytical simulations for a 200 -element and a 900 -element linear RRS, are depicted in Fig. 4, for 1-, 1.5-, and 2-bit quantization schemes. It is observed that the range of randomization required for minimum SLL should be at least 
$\left[0,180^{\circ}\right]$ for 1 -bit quantization, $\left[0,120^{\circ}\right]$ for 1.5 -bit, and $\left[0,90^{\circ}\right]$ for 2-bit, as summarized in Table II. This range of randomization also guarantees the lowest SLL achievable for each configuration. Moreover, it is noticed from the radiation patterns of Fig. 3 that the average SLL is also impacted by the random phase delays. Namely, as randomization is increased, the average SLL also increases. This is attributed to the random redistribution of the quantization lobe power to other directions.

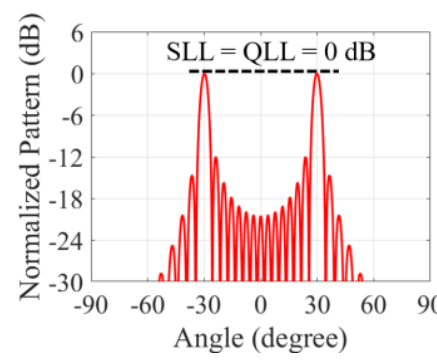

(a)

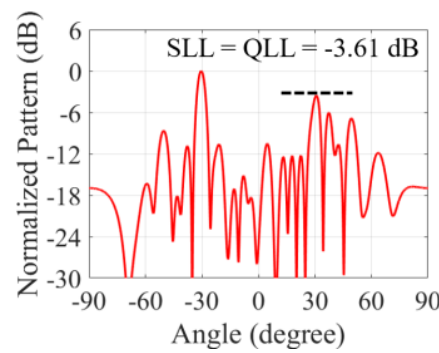

(c)

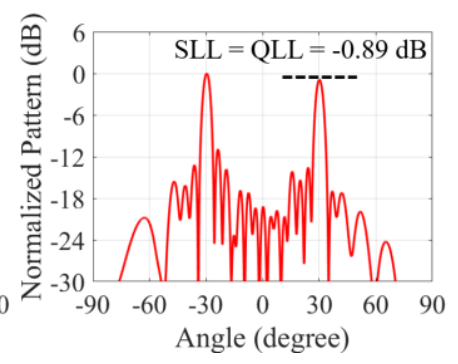

(b)

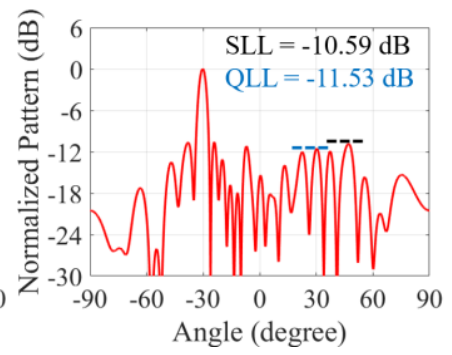

(d)
Fig. 3. Normalized radiation patterns of 30-element (linear) 1-bit reflective surface with (a) $0^{\circ}$ (no randomization), (b) randomization range of $0-60^{\circ}$, (c) $0-120^{\circ}$, and (d) $0-180^{\circ}$.

TABLE I

SIDE LOBE LEVEL V/S PHASE RANDOMIZATION

\begin{tabular}{|c|c|c|c|c|}
\hline \multirow[b]{2}{*}{$\begin{array}{l}\text { Test RRS / Randomization } \\
\text { range }\end{array}$} & \multicolumn{4}{|c|}{ Sidelobe level of 1-bit RRS (dB) } \\
\hline & $\mathbf{0}$ & 0 to $60^{\circ}$ & 0 to $120^{\circ}$ & 0 to $180^{\circ}$ \\
\hline 30-element Linear RRS & 0 & -0.89 & -3.61 & -10.59 \\
\hline 200-element Linear RRS & 0 & -0.56 & -7.37 & -11.43 \\
\hline 900-element $(30 \times 30) 2 D$ RRS & 0 & -0.81 & -6.03 & -15.38 \\
\hline
\end{tabular}

(a)

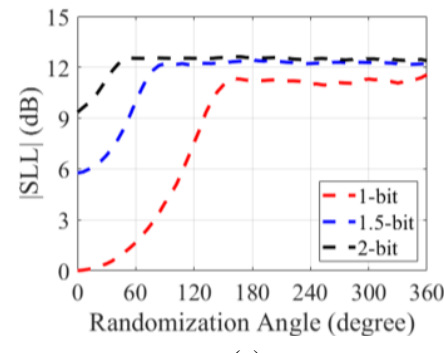

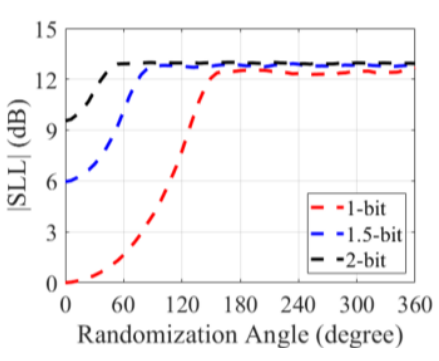

(b)
Fig. 4. Variation of the $|\mathrm{SLL}| \mathrm{v} / \mathrm{s}$ the range of randomization for (a) 200-element linear RRS and (b) 900-element linear RRS.

TABLE II

REQUiRED RANGe OF PHASE DELAy RANDOMIZATION ( $\Phi_{\text {rand_range }}$ ) FOR VARIOUS QUANTIZATION SCHEMES

\begin{tabular}{cc}
\hline Quantization scheme & $\boldsymbol{\Phi}_{\text {rand_range }}$ \\
\hline 1-bit & 0 to $180^{\circ}$ \\
1.5-bit & 0 to $120^{\circ}$ \\
2-bit & 0 to $90^{\circ}$ \\
\hline \hline
\end{tabular}

\section{REFLECTIVE SURFACE DESIGN}

In this section, we describe the design procedure for the $30 \times 30$ 2D RRS. A single layer design is considered for less fabrication complexity and reduced losses arising from the use of multilayered structure, both of which are typically exacerbated at $\mathrm{mmWave} / \mathrm{THz}$ frequencies.

\section{A. Unit-cell}

The architecture of the proposed randomized RRS is shown in Fig. 1b. Its unit-cell consists of an additional randomized delay phase along with the antenna and the switch. The delays are implemented through microstrip lines. The corresponding implementation of the single bit unit-cell proposed in this research is depicted in Fig. 5. The patch antenna is linearly polarized, and at the non-radiating edge, a transmission line feed with an embedded switch is integrated. The feed position $y_{0}=40 \mu \mathrm{m}$ is optimized to offer maximum signal coupling between the antenna and the transmission line. We choose the non-radiating side for feeding the antenna to minimize the metal structure footprint as well as to facilitate the development of higher-bit RRS designs in the future [22]. The dimensions of the patch are $284 \mu \mathrm{m} \times 185 \mu \mathrm{m}$, and it is designed to resonate at $222.5 \mathrm{GHz}$. By varying the length of the feed line branch $\Delta l$, the necessary random phase delays can be implemented for each unit-cell.

The switch is implemented in a coplanar waveguide (CPW) [23], as shown in the inset of Fig. 5. This configuration is favorable in terms of RF performance for switches that utilize planar, tunable 2D materials (e.g., graphene), as discussed in [24]. Herein, to demonstrate the proof-of-concept of the proposed technique, we use open and short transmission line terminations to emulate the on/off states of ideal switches. To maintain a planar topology and avoid the use of complex and high inductance vias, a microstrip to CPW transition is employed. The total size of the unit-cell is $665 \mu \mathrm{m} \times 665 \mu \mathrm{m}$, which is approximately $\lambda_{0} / 2$ at $222.5 \mathrm{GHz}$.

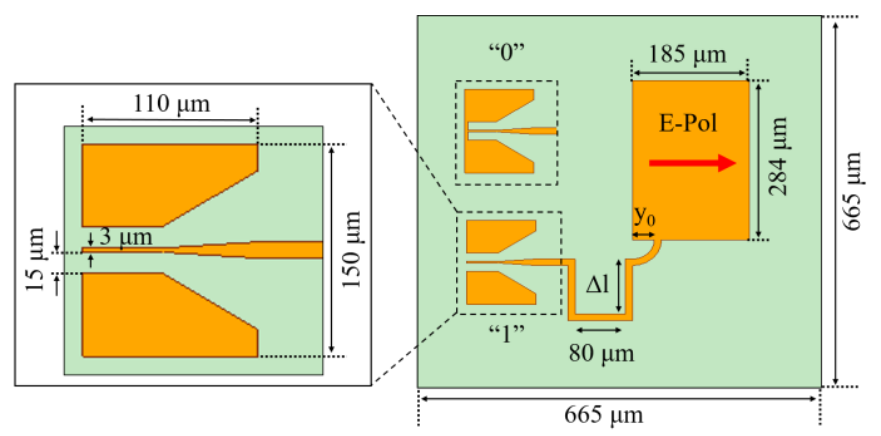

Fig. 5. A unit-cell of the designed reflective surface.

\section{B. 2D Reflective Surface Design}

In this section, both randomized and non-randomized RRS designs are compared through numerical analysis. For the nonrandomized case, $\Phi_{M \times N}^{r a n d}=0$, and thus, the phase delays are zero, as shown in Fig. 6a. Consequently, the corresponding continuous element phase obtained from (1) and shown in Fig. $6 \mathrm{~b}$ results in a repetitive quantized coding scheme. This repetitive coding scheme is caused by the periodicity of the phase rounding quantization error which makes the total phase, 
$\Phi_{M \times N}^{\text {total }}$ periodic, as shown in Fig. 6c. The resulting radiation pattern has both the desired main lobe along the $-30^{\circ}$ and an undesired quantization lobe along $+30^{\circ}$, as shown in Fig. $6 \mathrm{~d}$. This quantization lobe has the same magnitude as the main lobe, and thus, the QLL is $0 \mathrm{~dB}$.

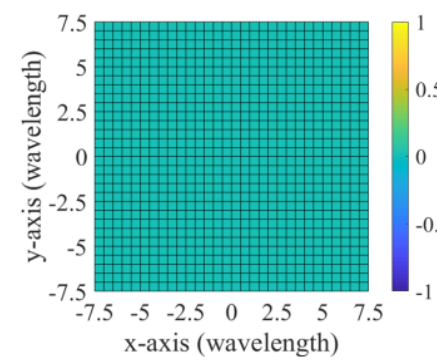

(a)

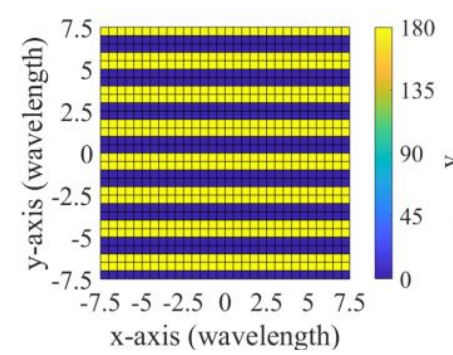

(c)

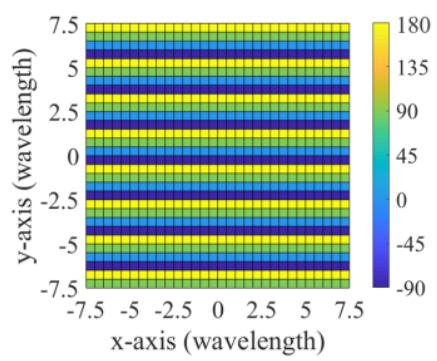

(b)

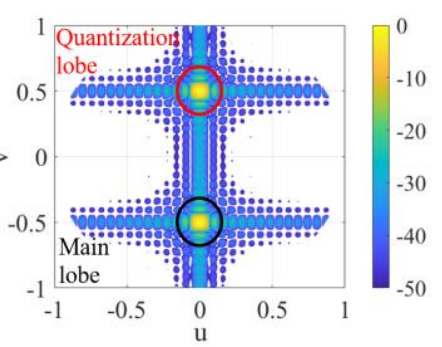

(d)
Fig. 6. $30 \times 302 \mathrm{D}$ non-randomized reflective surface design: (a) delay phase $\Phi_{30 \times 30}^{\text {rand }}=0$, (b) excitation phase $\Phi_{30 \times 30}$, (c) total phase $\Phi_{30 \times 30}^{\text {total based on }}$ 1 -bit quantization scheme, and (d) $3 \mathrm{D}$ radiation pattern in the $u$-v plane.

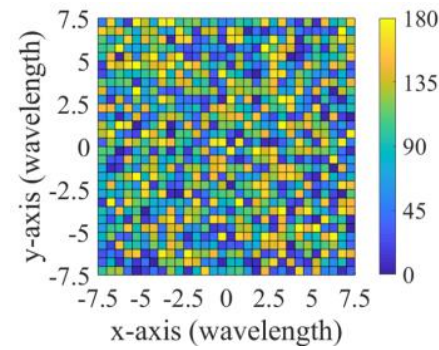

(a)

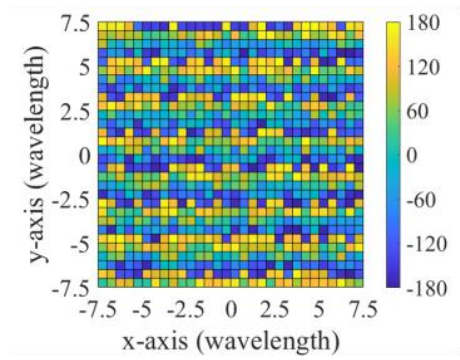

(c)

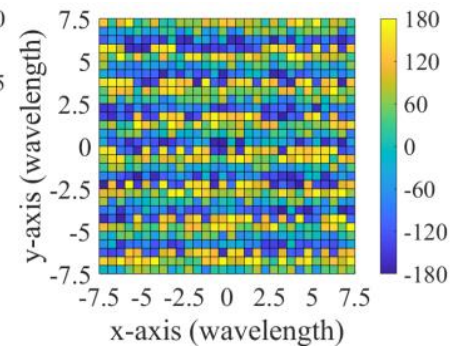

(b)

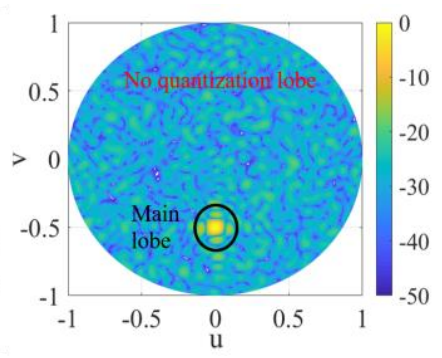

(d)
Fig. 7. 30×30 2D randomized reflective surface design: (a) randomly generated phase delays $\Phi_{30 \times 30}^{\text {rand }}$, (b) excitation phase $\Phi_{30 \times 30}$ obtained by adding random phase delays, (c) total phase $\Phi_{30 \times 30}^{\text {total }}$ based on 1-bit quantization scheme, and (d) 3D radiation pattern in the $u-v$ plane.

In contrast, for the case of randomized RRSs, $\Phi_{M \times N}^{\text {rand }} \neq 0$, the pseudorandom phase delays are generated from (5) and given in Fig. 7a. These phase delays correspond to the physical lengths of the microstrip feed line, $\Delta l$. Using (4), the calculated continuous element phases are shown in Fig. 7b. Consequently, the error periodicity of the corresponding total phase, $\Phi_{M \times N}^{\text {total }}$ is perturbed as shown in Fig. 7c, resulting in a single main lobe at $-30^{\circ}$, as depicted in Fig. 7d. Finally, the 2D power patterns in the $\phi=90^{\circ}$ elevation plane of Fig. 8 compares the two RRS topologies where it is observed that the random phase delay scheme eliminates the QLL.

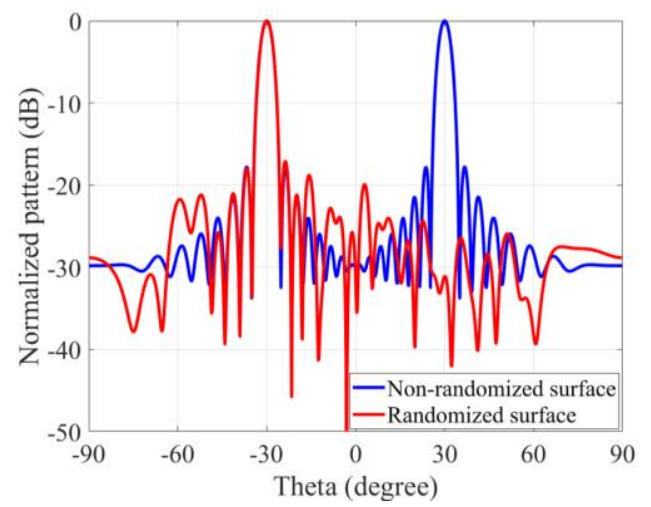

Fig. 8. Normalized 2D radiation pattern of the $30 \times 30$ reflective surface in the $\phi=90^{\circ}$ plane, comparing the designs with no randomization and full randomization $\left(0-180^{\circ}\right)$.

\section{SUB-MMWAVE REFLECTIVE SURFACE FABRICATION}

In this section, we detail the process used for the fabrication of the two single-layer $30 \times 30$ planar 1-bit RRSs, one employing full phase randomization $\left(0-180^{\circ}\right)$ and the other employing no randomization. The single-layer implementation reduces the fabrication complexities when compared to the existing methodologies used for fabricating reflective surfaces that produce single beam radiation patterns.

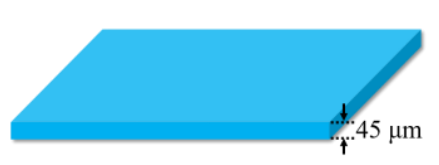

(a) Alumina ribbon ceramic wafer.

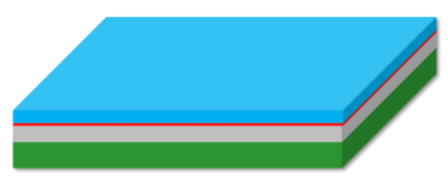

(c) Bonding to carrier wafer.

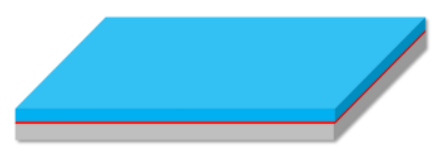

(b) Metal deposition.

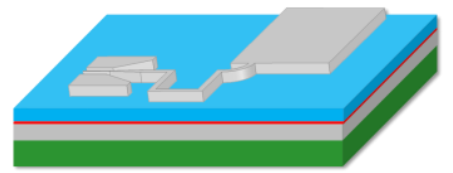

(d) Patterning + Lift-off.
Alumina ribbon ceramic substrate from Corning Inc.

Aluminum (ground + antenna)

Titanium

Carrier wafer (500 $\mu \mathrm{m}$ quartz)

Fig. 9. Fabrication process flow for the on-wafer development of the proposed reflective surface (a) $45 \mu \mathrm{m}$ thick, 2 " diameter alumina ribbon ceramic substrate used as the base wafer in the fabrication, (b) Ti/Al deposition to form the ground plane for the reflective surfaces, (c) $500 \mu \mathrm{m}$ thick, 2" diameter quartz wafer bonded to the metalized side of the wafer to impart sturdiness to the alumina ribbon ceramic wafer, and (d) device formation through spin coating, photolithography, patterning, and lift-off processes.

Both the non-randomized and the randomized RRSs are fabricated side-by-side on a $2 "$ diameter, $45 \mu \mathrm{m}$ thick alumina ribbon ceramic wafer from Corning [25]. The wafer has an $\epsilon_{r}=$ 10 and $\tan \delta=0.8 \times 10^{-3}$ obtained from quasi-optical reflectometer measurements at ASU. The alumina ribbon ceramic wafer is semi-transparent and flexible owing to its fine 
grain, dense microstructure and low thickness of $45 \mu \mathrm{m}$, so it was bonded to a $2 "$ diameter, $500 \mu \mathrm{m}$ thick quartz wafer to impart the sturdiness required by the alumina wafer for further fabrication steps. Initially, a $30 \mathrm{~nm}$ thick Ti layer followed by a $300 \mathrm{~nm}$ thick Al layer was deposited on one side of the alumina ribbon ceramic wafer. This layer serves as the ground plane for the radiating structures. Then the metalized side of the alumina ribbon ceramic wafer was permanently bonded with the carrier quartz wafer. The bonding process was carried out in the Flexible Electronic Display Center (FEDC) at ASU.

The bonded wafer was spin-coated with photoresists and exposed to UV through the photolithography step to develop the antenna patterns. Then a second metallization step is used to deposit $\mathrm{Al}$ for radiating structures, followed by a lift-off process, to obtain the final patterns as shown in Fig. 9d. The steps used in the fabrication process are illustrated in Fig. 9. In Fig. 10a we depict the finished alumina ribbon ceramic wafer with fabricated reflective surfaces. The randomized reflective surface is on the left, and the non-randomized reflective surface is on the right. A high-magnification image of the individual unit-cell is shown in Fig. 10b.

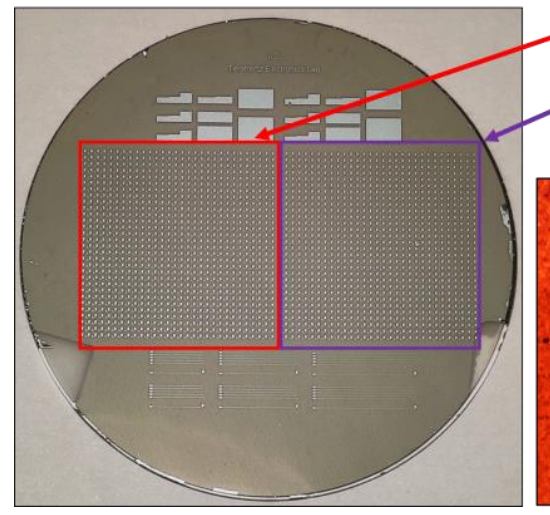

(a)
Randomized reflective surface reflective surface

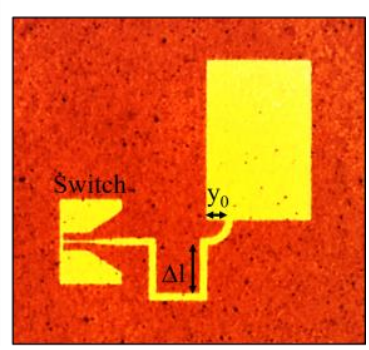

(b) Non-Randomized

Fig. 10. Fabricated alumina ribbon ceramic wafer with (a) randomized (left) and non-randomized RRS sections, and (b) high-magnification image of a unitcell of the fabricated RRSs.

\section{SUB-MMWAVE RCS CHARACTERIZATION}

A quasi-optical measurement setup is used to measure the RCS characteristics of the fabricated RRS prototypes. The setup represented in Fig. 11 consists of two vector network analyzer (VNA) extenders, a transmitting, and a receiving horn antenna, 2 collimating lenses, and the prototype wafer, all mounted on an optical breadboard. The signal from the VNA is upconverted to $222.5 \mathrm{GHz}$ using the VNA extender on the transmitter side and focused on the wafer. The Teflon lens in front of the transmitting horn antenna is used to collimate the diverging beam from the horn and provides an illumination beam of radius $1 \mathrm{~cm}$ on the reflective surfaces. The wafer is mounted on a sliding pedestal so that the RRS under test is aligned with the transmitter beam at boresight $\left(\theta_{i}=0\right)$. To measure the scattered fields, the receiver is rotated radially around the center of each RRS covering the $\left[-20^{\circ},-80^{\circ}\right]$ and $\left[+20^{\circ},+80^{\circ}\right]$ angle ranges, only restricted by the geometrical limitations of the setup. Nevertheless, a single point RCS measurement at $\theta_{d}=0^{0}$ (monostatic) is carried out to ensure that there is no significant reflection in the broadside direction.
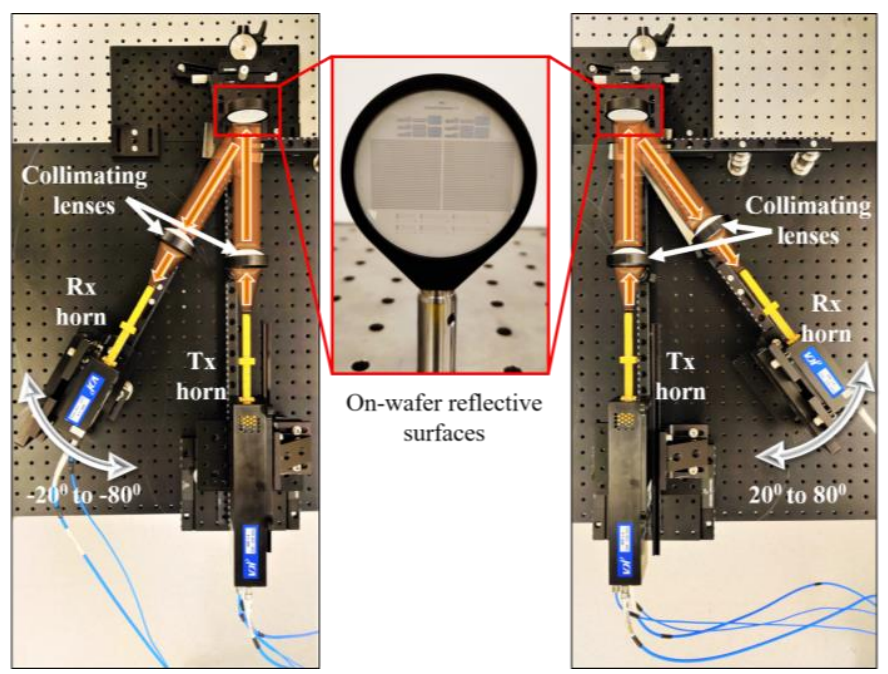

Fig. 11. Quasi-optical measurement setup used for the characterization of the fabricated reflective surfaces.

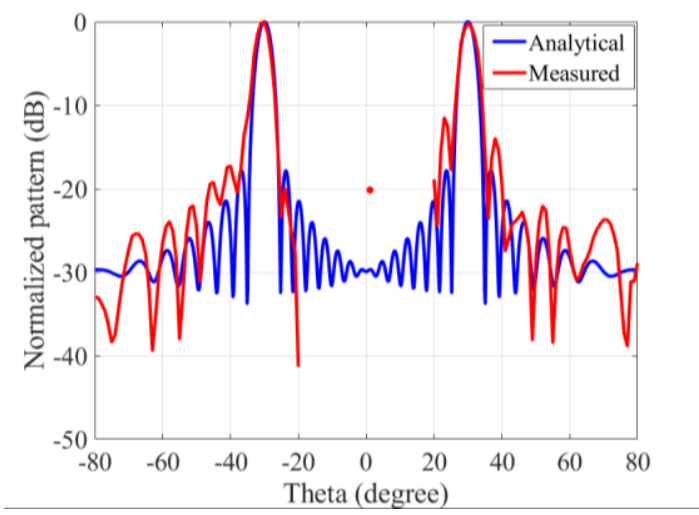

(a)

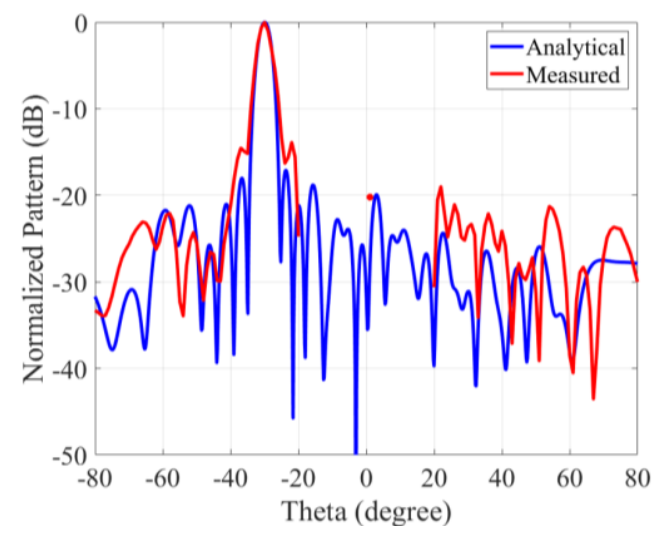

(b)

Fig. 12. Normalized RCS as a function of the scan angle theta at $222.5 \mathrm{GHz}$ for (a) non-randomized RRS and (b) randomized RRS.

The measured RCS patterns of the non-randomized and randomized RRSs at $222.5 \mathrm{GHz}$ have been plotted along with the analytical results, for both the surfaces in Fig. 12a and b, respectively. A good agreement is achieved between the analytical and measured results. The non-randomized RRS radiates at both $-30^{\circ}$ and $+30^{\circ}$, indicating the presence of an undesired quantization lobe, while the randomized RRS radiates only at $-30^{0}$, which confirms that the quantization lobe is eliminated by incorporating of phase randomization in the 
feed network of the RRS unit-cells. From the measurements, a QLL below $-18 \mathrm{~dB}$ is achieved.

Furthermore, to evaluate the bandwidth of operation, the RCS is plotted as a function of frequency and scan angle theta, as shown in Fig. 13a and b. For both non-randomized and randomized RRSs, the $3-\mathrm{dB}$ bandwidth is approximately 8.5 $\mathrm{GHz}$, which corresponds to a fractional bandwidth of $3.8 \%$.

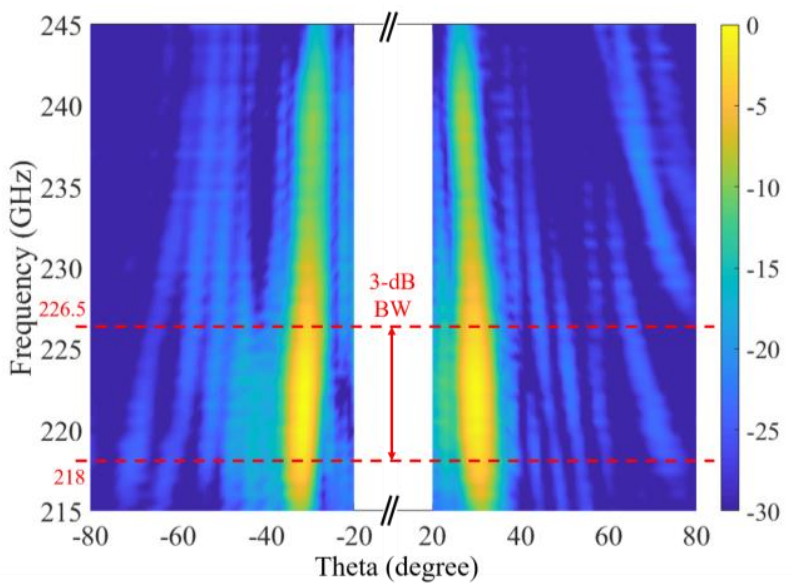

(a)

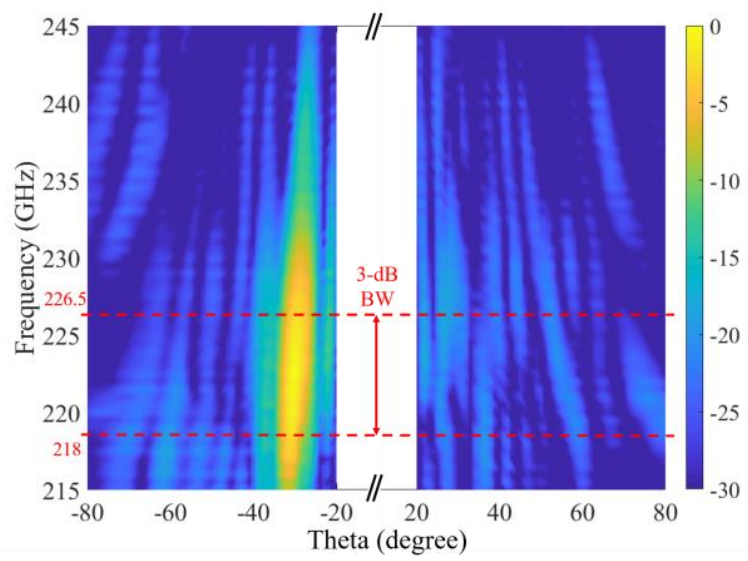

(b)

Fig. 13. Normalized RCS as a function of frequency and scan angle theta for (a) non-randomized RRS and (b) randomized RRS.

\section{DISCUSSION ON EFFICIENCY}

In this section, we discuss the losses encountered with the implementation of the 1-bit RRS prototype. As such, we measure the $S_{21}$ parameter at the second port of the VNA at the maximum reflection angle $\theta=-30^{\circ}$ for both non-randomized and randomized RRSs under broadside illumination, as depicted in Fig. 14. To account for the setup's measurement losses, the measured $S_{21}$ is normalized to a monostatic reflection measurement from an aluminum-coated wafer.

At $222.5 \mathrm{GHz}$, the RCS of the aluminum mirror is $-3.9 \mathrm{~dB}$ and is attributed to the discrepancies in the alignment and mirror planarity as well as losses from the lenses. Additional signal losses are listed below:

1. Quantization Loss: It results from the 1-bit quantization scheme employed in this research. It contributes to an additional loss of $-3 \mathrm{~dB}$.

2. Aperture Loss: It results from the choice of the main beam direction. Since $\theta=-30^{0}$ is chosen as the main beam direction, we have an aperture loss of $20 \log _{10} \cos (-30)=$ $-1.25 d B$.

3. Surface Waves Loss: It is due to the coupling between the antenna elements and the surface waves excited in the alumina substrate. To characterize this loss, we carry out full-wave simulations of the 1-bit RRS with no conductor and dielectric losses and compare it with the full-wave results of an ideal 1-bit RRS, which is modeled using PECPMC boundaries as unit-cells $(1.8 \mathrm{~dB})$.

4. Dielectric Loss: Free-space measurements using a quasioptical reflectometer setup estimated the loss tangent of alumina ribbon ceramic wafer as $\tan \delta=0.8 \times 10^{-3}$. Fullwave simulations show that the dielectric loss contributes an additional $0.17 \mathrm{~dB}$ of loss.

5. Conductor Loss: The losses due to the finite conductivity and roughness of aluminum metallization result in an estimated $3.37 \mathrm{~dB}$ loss.

To estimate the prototype's efficiency, we account for the loss mechanisms not present in an ideal 1-bit reflective surface comprised of PMC and PEC unit-cells designed for beam deflection at $\theta=-30^{\circ}$, namely, we consider surface wave, dielectric, and conductor losses. These losses summing up to $5.34 \mathrm{~dB}$ are listed in Table III and result in an efficiency of $30 \%$.

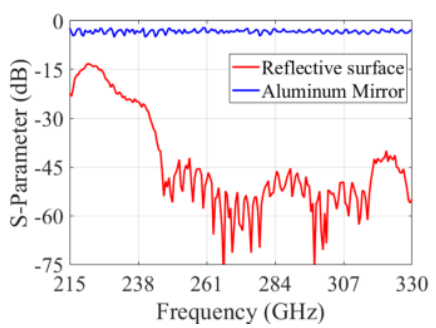

(a)

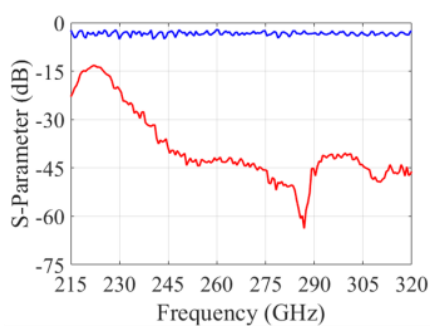

(b)
Fig. 14. RCS as a function of frequency at $\theta=-30^{\circ}$ for (a) non-randomized RRS and (b) randomized RRS.

TABLE III

BREAKDOWN OF ESTIMATED LOSSES (COMPARED TO AN IDEAL 1-BIT RRS)

\begin{tabular}{lc}
\hline \hline Source of loss & Magnitude of loss (dB) \\
\hline Surface Wave loss & -1.80 \\
Dielectric loss & -0.17 \\
Conductor loss & -3.37 \\
\hline Total loss & -5.34 \\
\hline \hline
\end{tabular}

\section{CONCLUSION}

We presented a robust technique for mitigating quantization lobes in sub-mmWave single-layer 1-bit RRSs under plane wave illumination using random phase delays. Specifically, the technique is realized by introducing randomized physical delay lines in the feed network of the individual unit-cells to break the periodicity of the quantization error and mitigate the quantization lobes. A comprehensive study explaining the effect of introducing varied degrees of randomization in a traditional RRS is presented along with the analytical results. Leveraging these results, we stipulate for the first time, the condition for choosing the range of randomization required to achieve the lowest SLL. Adopting the stipulated range of randomization, we have designed a $30 \times 30$ single-layer 1-bit randomized RRS at $222.5 \mathrm{GHz}$ to radiate along $-30^{\circ}$ direction. We have also designed a non-randomized RRS with similar 
specifications to carry out a comparative analysis. Both surfaces are fabricated side-by-side on a 2" diameter, $45 \mu \mathrm{m}$ thick alumina ribbon ceramic substrate from Corning Inc. The RCS of the fabricated reflective surfaces is characterized by a quasioptical measurement setup, and the results confirm that the quantization lobes in randomized RRS are suppressed with a QLL below -18 dB. To demonstrate the proof-of-concept of the proposed technique, the fabricated prototype is implemented with permanent, ideal switches using open and short terminations. Future work will focus on realizing the proposed topology with switches using tunable planar materials (e.g., graphene, vanadium dioxide).

\section{ACKNOWLEDGMENT}

For their valuable contribution to this work, we would like to acknowledge the use of facilities within the ASU NanoFab supported in part by the NSF program (No. NNCI-ECCS1542160). We would also like to thank Corning Inc. for providing the alumina ribbon ceramic substrates and fruitful discussions on handling and fabrication.

\section{REFERENCES}

[1] T. S. Rappaport et al., "Wireless Communications and Applications Above $100 \mathrm{GHz}$ : Opportunities and Challenges for 6G and Beyond," IEEE Access, vol. 7, pp. 78729-78757, 2019.

[2] M. H. Dahri, M. H. Jamaluddin, M. I. Abbasi, and M. R. Kamarudin, "A Review of Wideband Reflectarray Antennas for 5G Communication Systems," in IEEE Access, vol. 5, pp. 17803-17815, 2017.

[3] G. C. Trichopoulos, H. L. Mosbacker, D. Burdette, and K. Sertel, "A Broadband Focal Plane Array Camera for Real-time $\mathrm{THz}$ Imaging Applications," IEEE Transactions on Antennas and Propagation, vol. 61, no. 4, pp. 1733-1740, April 2013.

[4] P. C. Theofanopoulos, M. Sakr, and G. C. Trichopoulos, "Multistatic Terahertz Imaging Using the Radon Transform," IEEE Transactions on Antennas and Propagation, vol. 67, no. 4, pp. 2700-2709, April 2019.

[5] P. C. Theofanopoulos and G. C. Trichopoulos, "A Novel Fingerprint Scanning Method Using Terahertz Imaging," 2018 IEEE International Symposium on Antennas and Propagation \& USNC/URSI National Radio Science Meeting, Boston, MA, 2018.

[6] S. Vakalis, L. Gong, Y. He, J. Papapolymerou and J. A. Nanzer, "Experimental Demonstration and Calibration of a 16-Element Active Incoherent Millimeter-Wave Imaging Array," in IEEE Transactions on Microwave Theory and Techniques, 2020.

[7] R. J. Mailloux, "Phased array theory and technology," Proceedings of the IEEE, vol. 70, no. 3, pp. 246-291, March 1982.

[8] V. Jamali, A. M. Tulino, G. Fischer, R. Muller, and R. Schober, "Scalable and Energy-Efficient Millimeter Massive MIMO Architectures: ReflectArray and Transmit-Array Antennas," ICC 2019 - 2019 IEEE International Conference on Communications (ICC), Shanghai, China, 2019, pp. 1-7.

[9] J. Huang and J.A. Encinar, Reflectarray Antennas, Piscataway, NJ; Hoboken, NJ: IEEE Press; Wiley, 2008.
[10] S. V. Hum and J. Perruisseau-Carrier, "Reconfigurable reflectarrays and array lenses for dynamic antenna beam control: A review," IEEE Trans. Antennas Propag., vol. 62, no. 1, pp. 183-198, 2014.

[11] H. Kamoda, T. Iwasaki, J. Tsumochi, T. Kuki, and O. Hashimoto, "60$\mathrm{GHz}$ Electronically Reconfigurable Large Reflectarray Using Single-Bit Phase Shifters," IEEE Transactions on Antennas and Propagation, vol. 59, no. 7, pp. 2524-2531, July 2011.

[12] P. Nayeri, F. Yang, and A. Z. Elsherbeni, "Beam-Scanning Reflectarray Antennas: A technical overview and state of the art.," IEEE Antennas and Propagation Magazine, vol. 57, no. 4, pp. 32-47, Aug. 2015.

[13] E. Carrasco and J.A. Encinar, "Reflectarray Antennas: A Review," Forum of Electromagnetic Research Methods and Application Technologies (FERMAT), vol. 16, Aug. 2016.

[14] Q. Wu and R. Zhang, "Intelligent Reflecting Surface Enhanced Wireless Network: Joint Active and Passive Beamforming Design," 2018 IEEE Global Communications Conference (GLOBECOM), Abu Dhabi, United Arab Emirates, pp. 1-6, 2018.

[15] C. Huang, A. Zappone, G. C. Alexandropoulos, M. Debbah, and C. Yuen, "Reconfigurable Intelligent Surfaces for Energy Efficiency in Wireless Communication," in IEEE Transactions on Wireless Communications, vol. 18 , no. 8, pp. 4157-4170, Aug. 2019.

[16] P. C. Theofanopoulos and G. C. Trichopoulos, "Toward Large-Scale Dynamically Reconfigurable Apertures Using Graphene," 2019 IEEE International Symposium on Antennas and Propagation and USNC-URSI Radio Science Meeting, Atlanta, GA, USA, 2019.

[17] S. Costanzo, F. Venneri, A. Raffo, and G. Di Massa, "Dual-Layer SingleVaractor Driven Reflectarray Cell for Broad-Band Beam-Steering and Frequency Tunable Applications," in IEEE Access, vol. 6, pp. 71793$71800,2018$.

[18] H. Yang et al., "A Study of Phase Quantization Effects for Reconfigurable Reflectarray Antennas," in IEEE Antennas and Wireless Propagation Letters, vol. 16, pp. 302-305, 2017.

[19] M. Smith and Y. Guo, "A comparison of methods for randomizing phase quantization errors in phased arrays," in IEEE Transactions on Antennas and Propagation, vol. 31, no. 6, pp. 821-828, November 1983.

[20] J. Yin, Q. Wu, Q. Lou, H. Wang, Z. N. Chen and W. Hong, "Single-Beam 1 Bit Reflective Metasurface Using Prephased Unit-cells for Normally Incident Plane Waves," in IEEE Transactions on Antennas and Propagation, vol. 68, no. 7, pp. 5496-5504, July 2020.

[21] P. C. Theofanopoulos and G. C. Trichopoulos, "Mitigating Quantization Lobes in Reconfigurable Reflective Surfaces," 2020 IEEE International Symposium on Antennas and Propagation and USNC-URSI Radio Science Meeting, Montreal, Quebec, Canada, 2020.

[22] P. C. Theofanopoulos and G. C. Trichopoulos, "A Novel 2-Bit Graphene Reconfigurable Reflectarray," 2020 IEEE International Symposium on Antennas and Propagation and USNC-URSI Radio Science Meeting, Montreal, Quebec, Canada, 2020.

[23] Guizhen Zheng, J. Papapolymerou and M. M. Tentzeris, "Wideband coplanar waveguide RF probe pad to microstrip transitions without via holes," in IEEE Microwave and Wireless Components Letters, vol. 13, no. 12, pp. 544-546, Dec. 2003.

[24] P. C. Theofanopoulos and G. C. Trichopoulos, "Modeling of SubMillimeter Wave Coplanar Waveguide Graphene Switches," 2019 IEEE International Symposium on Antennas and Propagation and USNC-URSI Radio Science Meeting, Atlanta, GA, USA, 2019.

[25] Zhuang et al., "Flexibility matters: High purity, thin, flexible alumina ribbon ceramic, Ceramic \& Glass Manufacturing 1(4)," ACerS Bulletin 99(7): pp. 54-58, 2020. 